\title{
Start-up rheometry of highly polydisperse magnetorheological fluids: experiments and simulations
}

Article

Accepted Version

Ruiz-López, J. A., Wang, Z. W., Fernández-Toledano, J. C., Hidalgo-Alvarez, R. and de Vicente, J. (2016) Start-up rheometry of highly polydisperse magnetorheological fluids: experiments and simulations. Rheologica Acta, 55 (3). pp. 245-256. ISSN 1435-1528 doi: https://doi.org/10.1007/s00397016-0914-y Available at https://centaur.reading.ac.uk/54004/

It is advisable to refer to the publisher's version if you intend to cite from the work. See Guidance on citing.

To link to this article DOI: http://dx.doi.org/10.1007/s00397-016-0914-y

Publisher: Springer

All outputs in CentAUR are protected by Intellectual Property Rights law, including copyright law. Copyright and IPR is retained by the creators or other copyright holders. Terms and conditions for use of this material are defined in the End User Agreement.

www.reading.ac.uk/centaur 
Central Archive at the University of Reading

Reading's research outputs online 


\title{
Start-up rheometry of highly \\ polydisperse magnetorheological fluids: \\ experiments and simulations
}

\author{
J. A. Ruiz-López, ${ }^{1}$ Z. W. Wang, ${ }^{2}$ J. C. Fernández-Toledano, ${ }^{1}$ \\ R. Hidalgo-Alvarez ${ }^{1}$ and J. de Vicente ${ }^{1}$ \\ ${ }^{1}$ Department of Applied Physics, Faculty of Sciences, University of Granada, 18071- \\ Granada, Spain - jvicente@ugr.es \\ ${ }^{2}$ School of Mathematical and Physical Sciences, University of Reading, Reading \\ RG6 6AX, UK - zuowei.wang@ reading.ac.uk
}

\section{Submitted to Rheological Acta}

\begin{abstract}
An extensive experimental and simulation study is carried out in conventional magnetorheological fluids formulated by dispersion of mixtures of carbonyl iron particles having different sizes in Newtonian carriers. Apparent yield stress data are reported for a wide range of polydispersity indexes $(\mathrm{PDI})$ from $\mathrm{PDI}=1.63$ to $\mathrm{PDI}=$ 3.31 , which for a log-normal distribution corresponds to the standard deviation ranging from $v=0.38$ to $v=0.76$. These results demonstrate that the effect of polydispersity is negligible in this range in spite of exhibiting very different microstructures. Experimental data in the magnetic saturation regime are in quantitative good agreement with particle-level simulations under the assumption of dipolar magnetostatic forces. The insensitivity of the yield stresses to the polydispersity can be understood from the interplay between the particle cluster size distribution and the packing density of particles inside the clusters.
\end{abstract}

\section{Keywords}

Magnetorheology, magnetorheological fluids, yield stress, polydispersity, particle size distribution, simulations. 


\section{INTRODUCTION}

Conventional magnetorheological (MR) fluids are dispersions of carbonyl iron microparticles in non-magnetic carriers. In the absence of magnetic fields, the dispersions exhibit a liquid-like behavior. However, upon the application of a large enough magnetic field $(\geq 10 \mathrm{kA} / \mathrm{m})$, the particles are magnetized and interact with each other to form elongated structures in the direction of the magnetic field. This results in a field-controllable increase in the viscosity and the eventual appearance of an apparent yield stress at appropriate particle loadings and field strengths (so-called MR effect) (Parthasarathy and Klingenberg 1996, Bossis et al. 2002, Gonzalvez et al. 2006, Park et al. 2010, de Vicente et al. 2011).

Enhancing the yield stress under external fields is a priority for commercial applications. In this sense, it is well known that increasing the particle concentration results in an increase of the yield stress under the field (on-state) (e.g. Segovia-Gutierrez et al. 2012), but it also gives place to a large off-state (no field) viscosity (Barnes 1989) and eventually leads to a reduction in the MR effect (Foister 1997). One way to increase the particle volume fraction without increasing the off-state shear viscosity is by using polydisperse MR fluids (i.e., dispersions of magnetizable particles with different sizes). In fact, polydisperse MR fluids inherently exhibit a lower off-state viscosity than monodisperse MR fluids due to the different particle packing characteristics; larger packing fractions are achieved with polydisperse systems. This means that using polydisperse MR fluids, the particle volume fraction can be increased, without increasing the off-state viscosity, hence developing a larger MR effect.

The effect of particle size in the case of monodisperse systems has been largely reported in the literature for MR fluids, ER fluids and inverse ferrofluids (Lemaire et al. 1995, Tan et al. 1999, de Gans et al. 2000, Trendler and Bose 2005); in general, larger particles exhibit a larger yield stress under the presence of magnetic fields (e.g. Lemaire et al. 1995, Foister 1997, Trendler and Bose 2005). On the contrary, the understanding of polydisperse MR fluids is still not complete. As a first approximation towards the full understanding of the effect of particle size polydispersity, in recent years, a number of studies have focused on bimodal distributions (i.e. mixtures of particles having only two different sizes) (Ahn and Klingenberg 1994, Weiss et al. 1999, Weiss et al. 2000, Bombard et al. 2005, Golden and Ulciny 2005, Song et al. 2009). Experiments and 
simulations demonstrate that it is possible to substantially increase the yield stress of an initially monodisperse MR fluid with the addition of a small amount of smaller particles (at the same total particle concentration), while simultaneously reducing the viscosity of the suspension (Foister 1997, Weiss et al. 1999). In most cases, particle packing arguments are employed to explain such an increase (e.g. Weiss et al. 2000 for MR fluids; e.g. See et al. 2002 for ER fluids). However, more recently, Kittipoomwong et al. 2005 proposed an alternative mechanism for the enhanced yield stress in bidisperse suspensions by using particle level simulations. Interestingly, microstructure analysis revealed that the enhanced stress transfer in bidisperse suspensions was not associated with an increase in particle packing. Instead, the enhanced yield stress was associated with the presence of more highly anisotropic clusters of large particles than observed in monodisperse suspensions.

A complete understanding of the effect of particle size distribution in the MR effect of sphere-based suspensions is still missing in the literature. Actually, there are very few papers involving a continuous size distribution. To the best of our knowledge, there are only three papers addressing this issue from the simulation side. Wang et al. 1997 reported a two-dimensional (2D) simulation study of ER fluids under the assumption of Gaussian distribution of particle sizes and negligible thermal and inertial terms. They introduced a local-field approximation to take into account the mutual polarization effects between the particles. Shear stresses were simulated for a constant shear rate value at different standard deviations of the Gaussian distribution (from $s=0$ to 3 ). It was found that the stresses first dropped quickly with increasing polydispersity and then gradually saturate as $\mathrm{s}>0.5$ (corresponding to PDI $>1.07$, considering that the particle sizes were limited in a range of within $50 \%$ from the mean size). The decrease in the shear stress was interpreted as a result of the formation of imperfect chain-like structures by particles of different sizes that are easier to break under shear than those formed by particles of uniform size. Recently, Sherman and Wereley 1997 carried out a comprehensive 3D simulation study under the assumption of log-normal distribution, again neglecting thermal and inertial terms. Their results demonstrate that as the particle distribution size parameter increases, particles tend to form more irregular structures and a $25 \%$ reduction in the shear stress at low Mason numbers is observed. More recently, Fernández-Toledano et al. 2015 carried out 3D Brownian dynamic simulations of MR fluids with PDI $=1.12$ (i.e., the standard deviation of log-normal distribution 
$v=0.2$ ). The results obtained were compared with purely monodisperse systems. The effect of polydispersity was very small and basically only noticeable in the yielding region: a slightly more abrupt decrease in viscosity was found in viscosity curves for polydisperse suspensions.

Also, very few papers have been published on the effect of a continuous size distribution in the MR performance from the experimental point of view. The reason for this is that generally polydispersity is achieved by mixing only two populations having different particle sizes at different proportions (e.g. See et al. 2002 for ER fluids; e.g. Bombard et al. 2005 for MR fluids) and in most cases the particle size ratio is either large or extremely large (Song et al. 2009, Wu and Conrad 1998). To the best of our knowledge, there is only one paper in the scientific literature that addresses a continuous distribution by mixing more than two systems (Chiriac and Stonian 1998). In their paper, Chiriac and Stonian 1998 carried out an experimental investigation to elucidate the effect of particle size distribution on MR effect. Distributions were obtained first by sieving commercial micrometric iron particles (Sigma-Aldrich) to obtain narrow distribution powders and then mixing the finer powders in order to obtain three batches with tailored size distributions. MR fluids investigated were formulated at a $10 \mathrm{vol} \%$ by mixing the powders in mineral oil. Unfortunately, although some changes were detected when measuring the MR response, the mean particle size changed among the batches prepared and this complicated the interpretation of their results.

In this work we carry out an extensive experimental study on the effect of particle size polydispersity in conventional MR fluids prepared by dispersion of mixtures of three varieties of carbonyl iron microparticles that only differ in size and having all other physical properties essentially the same (chemical composition and magnetic properties). Particle concentrations and size distributions explored are within the range of those of interest in commercial applications (clearly larger than that reported by FernándezToledano et al. 2015). Special care is taken for the mean particle size of the distributions to remain constant (variations less than $5 \%$ ). Also, experimental results are compared to particle-level simulation data. 


\section{EXPERIMENTAL}

Three types of carbonyl iron particles were obtained from BASF SE and used without further purification (grades HQ, HS and OM). These three powders were conveniently mixed to further produce mixed particle systems with different particle size distribution and polydispersity but similar mean size and magnetic properties. MR fluids were prepared by dispersion of the carbonyl iron powders in a silicone (PDMS) oil of viscosity $20 \mathrm{mPa} \cdot \mathrm{s}$ (Sigma-Aldrich). The particle concentration was fixed at $10 \mathrm{vol} \%$. Table 1 summarizes relevant information on the polydispersity and magnetic properties of the iron grades and mixtures used in this manuscript. Magnetic properties of carbonyl iron powders were modelled using the Fröhlich-Kenelly law (Jiles 1991). Figure 1 contains the particle size distributions for the different MR suspensions investigated.

A MCR302 magnetorheometer (Anton Paar, MRD70/1T) was used in plate-plate configuration (20 mm diameter and 300 microns gap thickness). The magnetic field applied was always perpendicular to the plates and the temperature was maintained at $25{ }^{\circ} \mathrm{C}$ during the test. Experiments were carried out in saturation $\left(H_{0}=885 \mathrm{kA} / \mathrm{m}\right)$ for better comparison with the simulation results where the dipolar approximation is assumed.

The rheological protocol was as follows. First the sample was preconditioned at a high constant shear rate $\left(100 \mathrm{~s}^{-1}\right)$ for a duration of $30 \mathrm{~s}$ to remove history effects. Next, the suspension was left to equilibrate at rest for $60 \mathrm{~s}$ under the presence of a magnetic field. Finally, the rheological test started. It consisted of a logarithmic stress ramp from 1000 $\mathrm{Pa}$ to $10000 \mathrm{~Pa}$. The logarithmic increase of the stress was done at a rate of 50 points/decade and the acquisition time was $5 \mathrm{~s}$. The static yield stress was obtained from the stress corresponding to a sudden change in shear rate in log-log stress versus shear rate representations. For the dynamic yield stress, a regression fit was carried out in linlin representation for the data points measured at the largest shear rates. All experimental data reported in this work were averages of at least three independent measurements with fresh new samples. As an example, the referred static and dynamic yield stress are shown for a typical rheogram $(v=0.76$ and $\phi=0.10)$ in the Supporting Material (Figure S1). 


\section{SIMULATION TECHNIQUES}

Molecular dynamic simulations were carried out in order to understand the microscopic mechanisms behind the performance of polydisperse MR fluids under shearing flow. The simulation method was an extension of the methodology developed by Klingenberg and coworkers (Parthasarathy and Klingenberg 1996, Ahn and Klingenberg 1994, Kittipoomwong et al. 2005) for polydisperse particles taking the expressions for the forces from a previous work by Fernandez-Toledano et al. 2015 The method concerns non-Brownian inertialess simulations. In general, this restriction can be easily accepted in the case of conventional MR fluids, since the so-called Lambda ratio (i.e. the ratio between the magnetostatic energy and the thermal energy) is generally large enough to safely neglect the thermal motion. MR fluids were thus modeled as $N=1000$ neutral buoyant particles in a continuous Newtonian medium. Hydrodynamic interactions were also neglected and the Stokes' law approximated the drag force. This approximation is generally adopted because of computational reasons. Also, a recent study by Lagger et al. 2015 demonstrated that hydrodynamic interactions can be safely neglected if the hydrodynamic stress is not the main contribution to the total stress. Considering these approximations, the equation of motion of a particle $i$, can be expressed as follows:

$$
\zeta_{i}\left(\frac{d \vec{r}_{i}}{d t}-\vec{u}_{i}^{\infty}\right)=\vec{F}_{i}
$$

where $\zeta_{i}=3 \pi \eta_{c} \sigma_{i}$ is the friction coefficient of the particle with $\eta_{c}$ the Newtonian medium viscosity and $\sigma_{i}$ the diameter of the particle, respectively. $\vec{r}_{i}$ is the position vector of the particle. $\vec{u}_{i}^{\infty}=\dot{\gamma}_{i} \widehat{e}_{x}$ is the ambient fluid velocity at the particle center with $\dot{\gamma}$ the magnitude of the shear rate tensor, $z_{i}$ the $z$-coordinate of the particle and $\widehat{e}_{x}$ the unit vector in the $x$-direction in Cartesian coordinates. Finally, $\vec{F}_{i}$ is the total force acting on the particle. 
In Equation [1], the term $\vec{F}_{i}$ includes the pair-wise magnetostatic forces exerted by all other particles on particle $i, \vec{F}_{i}^{m a g}=\sum_{j \neq i} \vec{F}_{i j}^{m a g}$. Magnetostatic interaction force between two particles was modeled in the dipolar approximation as follows:

$$
\vec{F}_{i j}{ }^{m a g}=F_{0} \frac{\sigma_{i}^{3} \sigma_{j}^{3}}{\sigma_{m}{ }^{2} r_{i j}{ }^{4}}\left[\left(3 \cos ^{2} \theta_{i j}-1\right) \hat{e}_{r}+\sin 2 \theta_{i j} \hat{e}_{\theta}\right]
$$

Here, $F_{0}=3 \pi \mu_{0} \mu_{c r} \beta^{2} H_{0}{ }^{2} \sigma_{m}{ }^{2} / 16$ stands for the typical magnetic interaction force between two particles with sizes of the average diameter $\sigma_{m} \cdot \mu_{0}$ is the magnetic permeability of the vacuum, $\mu_{c r}$ is the relative permeability of the continuous medium, $\beta=\left(\mu_{p r}-\mu_{c r}\right) /\left(\mu_{p r}+2 \mu_{c r}\right)$ is the so-called contrast factor (or coupling parameter), $\mu_{p r}$ is the magnetic permeability of the particles, $H_{0}$ is the external magnetic field strength, $r_{i j}$ is the center-to-center distance between two particles $i$ and $j, \theta_{i j}$ is the angle between the line joining the centers of the two particles and the magnetic field direction, and $\hat{e}_{r}$ and $\hat{e}_{\theta}$ are the unit vectors in the directions $r$ and $\theta$ using a spherical coordinate system.

Exponential short-range repulsive forces were included to avoid overlapping between particles, $\vec{F}_{i j}^{\text {rep }}$, and between each particle and the two confining walls, $\vec{F}_{i}^{\text {wall,top }}$ and $\vec{F}_{i}^{\text {wall,bottom }}$. Exponential forces are applied instead of stiff power law forces, since it was shown that exponential forces help the formation of thicker aggregates as already observed in experiments (Melrose and Heyes 1993, Segovia-Gutiérrez et al. 2013). Expressions for the exponential forces used in this manuscript for continuous particle size distributions can be found in the work of Fernández-Toledano et al. (2015).

The equation of motion was made dimensionless using the following units: $l_{s}=\sigma_{m}$, $F_{s}=F_{0}$ and $t_{s}=3 \pi \eta_{c} \sigma_{m}{ }^{2} / F_{0}$. In simulations of continuously polydisperse systems we used a Log-normal distribution. The Log-normal distribution is a continuous probability 
distribution where the logarithm of the random variable is normally distributed. The Log-normal random variable was obtained, thus, by first calculating a normal variable of mean $\ln \sigma_{m}$ and standard deviation $v$ and then calculating the Log-normal random variable by taking the exponential of the normally distributed variable. Simulations also involved tri-disperse particle size distributions (i.e., mixtures of particles with three different sizes) to better understand the relationship between the field-induced microstructures and the rheological performance of the polydisperse MR fluids. Tridisperse particle sizes, $\sigma_{s}<\sigma_{a}<\sigma_{b}$, for each given polydispersity index were obtained by taking three different average diameters from the Log-normal distribution with fixed probabilities of $P\left(\sigma_{s}\right)=P\left(\sigma_{b}\right)=0.16$ and $P\left(\sigma_{a}\right)=0.68$. Table 2 summarizes tridisperse diameters as a function of the standard deviation, $v$, and so correspondingly the polydispersity index. From now on, we will also refer $v$ as the polydispersity index.

Once the total force acting on a particle was calculated, its equation of motion was solved using the Euler algorithm. Time variation was calculated at every step such that the maximum displacement of any particle, $i$, in one direction was no larger than $0.05 \sigma_{i}$ in order to avoid particles to be ejected from the box due to the fact that bigsmall particle interactions can provoke the smallest particle to move outside the box. This condition typically reduced the time variation and the total simulation time increases specially for the highest polydispersity indexes.

Stress growth tests (start-up tests) were carried out at a small-normalized shear rate, $\dot{\gamma}^{*}=10^{-3}$. This velocity was found to provide a stress value close to the yield stress (Fernández-Toledano et al. 2015). Simulations were composed of three stages: (i) Particles were randomly distributed in the simulation box, (ii) Particles were allowed to move under the presence of the magnetic field in quiescent state until reaching a stationary state. The stationary state was found to be established for all polydispersity indexes at a dimensionless time $t_{\text {stat }}{ }^{*}=3000$; (iii) Start-up test was properly initiated and steady shear flow was fully achieved when reaching a total strain of $\gamma=3$. The stress tensor was calculated at each time step as follows: 


$$
\tau_{\alpha \beta}=-\frac{1}{V} \sum_{i \neq j} r_{i j}^{\alpha} F_{i j}^{\beta}
$$

where $\tau_{\alpha \beta}$ is the $\alpha$ - $\beta$-component of the stress tensor, $V$ is the volume of the simulation box, $r_{i j}^{\alpha}$ is the $\alpha$-component of the distance between particles $i$ and $j$ and $F_{i j}^{\beta}$ is the $\beta$ component of the total pair-wise interaction between the two particles. Three regions were clearly identified in the stress versus time (or strain) curves: elastic, peak and steady regions (Fernández-Toledano et al. 2015). The elastic region corresponds to the low-strain regime where the stress is found to be proportional to the shear strain. Then, a maximum in the stress (peak) is observed. Finally, for large enough strain values the stress levels off to a nearly constant value as a function of time in a steady region. As the shear rates imposed were very low, the static yield stress can be assimilated as the stress peak. Also, the dynamic yield stress was calculated as the shear stress averaged over 4000 configurations saved from strain $\gamma=2$ to $\gamma=3$. Simulation results were the average values of at least 3 different tests for each case. In order to obtain a better estimation of the static yield stress, more simulation runs (at least 10) were carried out up to a reduced total strain of $\gamma=1$.

To better understand the rheological performance of polydisperse MR fluids, we ran a microstructural analysis through the calculation of the particle pair distribution function. In the canonical ensemble, the probability of finding a pair of particles at positions $\vec{r}_{1}$ and $\vec{r}_{2}$ respectively is given by the pair distribution function:

$$
g_{2}\left(\vec{r}_{1}, \vec{r}_{2}\right)=\frac{N(N-1)}{\rho^{2} Z_{N V T}} \int d \vec{r}_{3} d \vec{r}_{4} \ldots d \vec{r}_{N} \exp \left[-U\left(\vec{r}_{1}, \vec{r}_{2}, \ldots, \vec{r}_{N}\right) / k_{B} T\right]
$$

where $\rho$ is the number density of the particles, $Z_{N V T}$ is the partition function, $U\left(\vec{r}_{1}, \vec{r}_{2}, \ldots, \vec{r}_{N}\right)$ is the total interaction potential, $k_{B}$ is the Boltzmann constant and $T$ is the temperature of the system. 
Due to the spherical and azimuthal symmetries in the pair dipolar magnetic interaction, Equation [4] could be reduced to a function of the radial distance between two particles, $r$, and the angle between the direction vector linking the centers of these two particles and the direction of the magnetic field, $\theta$. Then the pair distribution function is converted to $g_{2}(r, \theta) \equiv g(r, \theta)$. In simulations, this function could be obtained simply by the following expression (Allen and Tildesley 1987):

$$
g(r, \theta)=\frac{V}{N^{2}}\left\langle\sum_{i} \sum_{j \neq i} \delta\left(r-r_{i j}\right) \delta\left(\theta-\theta_{i j}\right)\right\rangle
$$

where the bracket refers to a volume average. Also, a radial distribution function could be easily obtained from the radial and angular distribution function by the integration of

this function as a function of $\theta: g(r)=\int_{0}^{\pi / 2} d \theta \sin (\theta) g(r, \theta)$.

\section{RESULTS AND DISCUSSION}

\section{Experimental yield stresses}

In Figure 2 we show the rheograms (steady-state shear stress versus shear rate curves) corresponding to MR fluids having different polydispersity levels (from $v=0.38$ to $v=0.76$ ) at particle volume fraction $\phi=0.10$. For low stress levels the shear rate remains below $10^{-3} \mathrm{~s}^{-1}$ suggesting that the sample is not flowing (data not shown in the figure). However, for stresses above 6000-7000 $\mathrm{Pa}$ the shear rate dramatically increases in accordance to the initiation of flow. As observed, the curves essentially overlap suggesting that the effect of polydispersity is not important within the standard deviation of the data.

These results are in qualitative agreement with the simulation work of FernándezToledano et al. 2015. In that work, a simulation study was carried out for both monoand polydisperse (with $v=0.2$ ) MR fluids. These two MR fluids exhibited very similar behavior, the only difference being in the transition region between the solid and liquidlike regimes. Actually, for polydisperse MR fluids a slightly sharper transition was found if compared to the monodisperse case. 
Figure 3 contains experimental (static and dynamic) yield stresses for a particle concentration of $\phi=0.10$. As observed, the yield stresses do not depend much on the polydispersity index. They remain basically constant over the range of $v$ values we studied, with the dynamic yield stress being higher than the static one (see Figure S1 for the measurement of the two stresses). Only a very slight local maximum in the yield stress was measured for a polydispersity index of $v \approx 0.7$. Experiments were also carried out for other particle concentrations including $\phi=0.01, \phi=0.05$ and $\phi=0.20$, all giving very similar results. These experiments are not shown for brevity. To get a better insight into the effect of polydispersity we pursued particle-level simulations.

\section{Simulated yield stresses for MR systems with continuous particle size distributions}

The static and dynamic yield stresses of the MR systems with continuous particle size distributions were determined using particle-level simulations. The start-up tests were performed at a low shear rate $\left(\dot{\gamma}^{*}=10^{-3}\right)$ similar to that used by Fernández-Toledano $e t$ al. 2015. As an example, typical stress-strain curves for $\phi=0.10$ and $v=0.64$ are presented in Figure 4 for a total of 13 independent runs for strains up to $\gamma=1$ ( 4 runs for strains up to $\gamma=3$ ) to appreciate the repeatability of the simulations. In general, three regions can be identified: a first elastic region where the stress grows linearly with strain, a maximum (peak) in the stress, and finally a monotonous decay of the stress towards a final steady region marked by the long-time plateau. On the one hand, the maximum of the stress in each stress-strain curve is taken here as an estimate for the static yield stress in the suspension. On the other hand, the long-time stress plateau is taken as the dynamic yield stress of the suspension. In this sense, the dynamic yield stress was obtained as the average of the stress values from $\gamma=2$ to $\gamma=3$. It is worthwhile to note that the high number of repetitions was necessary to obtain a good estimation for the static yield stress (more than 10 independent runs). In the case of the dynamic yield stress, as it was already taken as a time average in each individual test, less (but at least 3 ) repetitions were needed to get a reasonably good estimation.

Simulated yield stresses are reported in Figure 5 for the particle concentration $\phi=0.10$. In good agreement with the experimental observations (c.f. Figure 3), both the static and dynamic yield stresses exhibit very minor changes with the variation of the degree of 
polydispersity. Despite many simplifications made in the simulation model, the simulation data fall well into the same quantitative range as the yield stresses measured in experiments. It is useful to remark that the definitions of the static and dynamic yield stresses differ between the experimental and simulation cases, as described in the previous sections. This difference, however, has no qualitative effect on the observed behavior of the yield stresses with respect to the level of polydispersity.

The mechanical properties of the MR fluids are inherently correlated to the microstructures formed by the particles. For convenience of structural analysis, we have also decided to carry out simulations on tri-disperse MR systems.

\section{Simulated yield stresses for MR systems with tri-disperse particle size distributions}

Simulation results on the static and dynamic yield stresses of the tri-disperse MR fluids are also contained in Figure 5. It can be seen that these stress values are very similar to those obtained from the MR systems with continuous size distributions, suggesting that the tri-disperse suspensions can closely capture the behavior of the experimental systems with continuous particle size distributions. Simulations of penta-disperse model systems have also performed. As expected, they provided consistent results (not shown for simplicity) with the case of tri-disperse suspensions, suggesting again that the tridisperse model suspension is sufficient to represent the continuous size distribution. We can then analyze the structural properties of these model systems for getting insights into the physical reasons of the negligible dependence of the yield stresses on the polydispersity.

Characterizing structural formation in simulated MR systems with continuous size distributions

To explore the structural characteristics of the particle aggregates formed in the MR systems, we calculated the average number of clusters $N_{c}$, the average number of particles in each cluster $N_{p c}=\sum_{i} n_{i} / N_{c}=N / N_{c}$ and the weight-averaged number of particles in each cluster $S_{2}=\sum_{i} n_{i}^{2} / \sum_{i} n_{i}=\sum_{i} n_{i}^{2} / N$ for the cases both prior to and under shear. Here, $n_{i}$ is the number of particles in the cluster $i$. The simulation results 
on $N_{p c}$ are shown in Figure 6, while those for $N_{c}$ and $S_{2}$ are given in Figures S2 and S3 in the Supporting Information. Note that there is a simple inverse relationship between $N_{c}$ and $N_{p c}$ because of the use of constant number of particles in the simulation box.

Simulation results in the absence of shear demonstrate a monotonic increase of $N_{c}$, and a concomitant decrease of $N_{p c}$ and $S_{2}$ with the increased polydispersity. For the highest polydispersities, the stationary state is difficult to achieve but the number of clusters only varies in less than 1 for reduced times higher than 2700. Considering the insensitivity of the yield stresses to the polydispersity, these simulation results reveal the importance of the internal microstructure of the aggregates for understanding the experimentally observed trends in the yield stress. They indicate that the yield stress depends not only on the number and sizes of the aggregates, but also on their mechanical strength to deformation. This is further supported by the simulation data upon shear, which show qualitatively similar structural changes with respect to polydispersity.

\section{Particle radial distribution function for MR systems with continuous particle size distributions}

The particle radial distribution functions can provide more detailed information about the particle packing inside the clusters. The $g(r)$ curves obtained from the MR systems with continuous particle size distributions are shown in Figure 7. These functions are calculated at three different stages of the deformation process: at $\gamma=0.1$ within the elastic region, at the stress peak, and in the steady regime from $\gamma=2$ to $\gamma=3$. As can be seen, all pair distribution functions exhibit a maximum (first peak) close to the average particle diameter. The width of the peak increases with increasing polydispersity, which is expected as a result of the connections between particles of a broader range of sizes. Interestingly, the height of this peak is also found to grow with the increased polydispersity. This means that on average each particle in the suspension with higher polydispersity finds more nearest neighbors than the particles in the less polydisperse suspensions. In other words, the particle packing density is higher in the former case. This phenomenon can be easily understood from the fact that small 
particles can get into the voids left by large particles in the clusters. The more heterogeneous packing of particles in the highly polydisperse systems leave only one peak in their $g(r)$ curves. On the contrary, the particle pair distribution functions of the less polydisperse systems possess a long-distance peak at reduced distance of $r^{*} \approx 5$ or 6. The long-distance peak is related to the mean distance between clusters since $g(r, \theta)$ is higher for angles from $\left[60^{\circ}, 90^{\circ}\right]$ that in angles from $\left[0^{\circ}, 30^{\circ}\right]$.

Comparing the simulation results in Figures 6 and 7, it can be seen that although the aggregates or clusters formed in the more polydisperse systems are of smaller sizes, the packing density of particles in these clusters are higher. Since the higher packing density may allow the clusters to sustain stronger mechanical load or deformation, this contribution could effectively cancel out the stress reduction effect caused by the decrease in cluster sizes. It is the interplay between the two opposite effects that leads to the nearly invariant yield stress upon changes of polydispersity.

The particle packing effect on the yield stress can be further examined by analyzing the angle-dependent pair distribution function $g(r, \theta)$ [Equation (5)]. Figure 8 presents the polydispersity dependence of the maximum (peak) value of the pair distribution function averaged within different angle limits, $g(r)_{\theta \in\left[\theta_{1}, \theta_{2}\right]}=\int_{\theta_{1}}^{\theta_{2}} d \theta \sin (\theta) g(r, \theta) / \int_{\theta_{1}}^{\theta_{2}} d \theta \sin (\theta)$. Two angle intervals are included. We show the simulation data for the (dipolar) energetic-favorable angles, $\theta \in\left[0,30^{\circ}\right]$ and for the energetic-unfavorable angles, $\theta \in\left[60^{\circ}, 90^{\circ}\right]$. Although in both cases the peak values show a monotonic increase with increasing polydispersity, the corresponding increases in the packing densities at different angles contribute to the total interaction energy and consequently the yield stress of the system in very different ways. The increase in the peak value of the pair distribution function at the energetic-favorable angles $\left(\theta \in\left[0,30^{\circ}\right]\right)$ suggests the formation of well-arranged and so stronger structures along the magnetic field direction. But the increase of the pair distribution function at the energeticunfavorable angles $\left(\theta \in\left[60^{\circ}, 90^{\circ}\right]\right)$ implies that the structures also contain more bonds that are easier to break. As will be seen below, it is not only the average local density of particles what contributes to the yield stress, but also the direction-dependent microscopic arrangements of the particles inside the clusters. It is also important to 
remark that the maximum value of the pair distribution function for the smallest angles decreases in the steady region compared with the elastic region, suggesting the break-up of the column- or chain-like structures along the field direction.

These results in Figure 8 are in agreement with the results from Fernandez-Toledano et al. 2015 (see Figure 4 in that work) where the particle connectivities in the polydisperse system demonstrated a continuous distribution as a function of the connecting angle, instead of individual peaks marking the more favorable connections in the monodisperse system, for $\theta \in\left[0,60^{\circ}\right]$. The dependence of particle connections on their sizes can be identified more easily for the tri-disperse systems, as shown below.

\section{Radial distribution function for tri-disperse MR systems}

In Figure 9 we show the radial distribution function curves for the tri-disperse MR suspensions. We observe that there are very reproducible peaks associated with the discrete particle sizes, namely the distances between particles of different sizes (small, average and big). In the case of the lowest polydispersity, another long-distance peak (at $\left.r^{*} \approx 5-6\right)$ is found, which does not occur in the highly polydisperse systems. This peak cannot be associated to the three different sizes of the particles, but can be well understood from microstructural snapshots shown in Figure 10 for two different polydispersities, $v=0.52$ and $v=0.72$. These snapshots demonstrate that bunches of average-size particles serve as bridges connecting big particles to from chain-like structures. As in the continuous case, for larger polydispersities, the pair distribution function and the interparticle connections suggest a stronger cluster formation, which implies a higher yield stress. However, these microstructural differences are not enough to provide a significantly higher yield stress, and so only a slight enhancement can be observed in experiments and simulations.

First row in Figure 11 contains the maximum (peak) values in the radial distribution functions shown in Figure 9 for different polydispersity indexes, taking into account all possible connections between particles of different sizes. At first sight, these figures suggest that the average-average (a-a) particle connections dominate the response. However, this could be misleading, because the probability of finding a big or small particle is much lower than the probability of finding an average-size particle. It is more 
helpful to obtain the relative importance of the connections among particles. In the simulations, we have $68.2 \%$ of average-size particles and only $15.9 \%$ of small-big particles, respectively. Hence, the results in the first row of Figure 11 were normalized by the probabilities of finding the different pairs of particles and presented in the second row of the figure. It then becomes evident that the small-small, small-big and big-big connections present a higher relative importance in determining the yield stress.

Similar results have been found in the radial distribution functions in the peak and steady shear regions (these results are included in Figure 11). Interestingly, the longdistance peak observed in the elastic region of the lowest polydisperse systems is not observed now in the peak and steady shear regions due to the breakage of large columnar structure. The heights of the peaks are also lower in the steady region than those found in the elastic region.

\section{CONCLUSIONS}

In this work we investigate the role of the particle size polydispersity using experimental and simulation start-up tests. Polydispersity index was varied in a wide range containing typical experimental polydispersities, from $\mathrm{PDI}=1.63$ to $\mathrm{PDI}=3.31$. In this PDI range, the effect of the polydispersity on the yield stress can be considered as negligible in experiments; only a very slight maximum can be observed in the experimental case for PDI $=2.88(v=0.64)$.

Two different particle size distributions were used in simulations: a continuous lognormal particle size distribution and a tri-disperse (i.e. three fixed different particle sizes) distribution. The results for the yield stress in both particle size distributions are in agreement with the experimental data and a slight but non-significant increase of the yield stress is found for the highest polydispersities.

Although variations in the yield stress were of minor importance, differences in the microscopic structures were found in simulations in the continuous and tri-disperse distributions. Analysis results on the particle cluster sizes and the particle radial distribution function show that increasing the level of polydispersity of the MR system leads to a smaller average number of particles per cluster but a higher packing density of the particles inside the clusters. Although the smaller cluster sizes may result in a 
reduction in the yield stress, the higher packing density can enhance the sustainability of the clusters to stronger mechanical load or deformation. It is the interplay between the two opposite effects that lead to the nearly negligible dependence of the yield stresses on the polydispersity.

Moreover detailed analysis of the angle-dependent pair distribution functions reveals that at high level of polydispersity particle connections are increased at all the angles with respect to the magnetic field direction (both energetic-favorable and energeticunfavorable directions). This suggests another effect that, although the structure gets denser with increasing polydispersity, the bonds between particles can become weaker. This also contributes to maintain nearly constant yield stress upon variation of polydispersity.

\section{ACKNOWLEDGEMENTS}

This work was supported by MAT 2013-44429-R project (Spain) and by Junta de Andalucía P10-RNM-6630, P10-FQM-5977 and P11-FQM-7074 projects (Spain). J.A.R.-L. acknowledges the financial support by the "Ministerio de Educación: Becas del Programa de Formación del Profesorado Universitario (FPU)” (AP2010-2144).

\section{REFERENCES}

Ahn KH; Klingenberg DJ (1994) Relaxation of polydisperse electrorheological suspensions. J. Rheol. 38(3):713-741.

Allen MP, Tildesley DJ (1987) Computer Simulation of Liquids. Oxford University Press, New York.

Barnes HA, Hutton JF, Walters K (1989) An Introduction to Rheology. Elsevier, Amsterdam.

Bombard AJF, Alcantara MR, Knobel M, Volpe PLO (2005) Experimental study of MR suspensions of carbonyl iron powders with different particle sizes. Int. J. Mod. Phys. B 19(07-09):1332-1338.

Bossis G, Volkova O, Lacis S, Meunier A (2002) Magnetorheology: Fluids, Structures and Rheology. In: Odenbach, S. (ed.) Ferrofluids. Springer, Berlin, pp 202-230.

Chiriac H, Stonian G (2009) Influence of particle size distributions on magnetorheological fluid performances. J. Phys. Conf. Ser. 200:072095. 
de Gans BJ, Duin NJ, van den Ende D, Mellema J (2000) The influence of particle size on the magnetorheological properties of an inverse ferrofluid. J. Chem. Phys. 113:2032-2042.

de Vicente J, Klingenberg DJ, Hidalgo-Álvarez R (2011) Magnetorheological fluids: a review. Soft Matter 7:3701-3710.

Fernández-Toledano JC, Ruiz-López JA, Hidalgo-Álvarez R, de Vicente J (2015) Simulations of Polydisperse Magnetorheological Fluids: a Structural and Kinetic Investigation. J. Rheol. 59(2):475-498.

Foister RT (1997) Magnetorheological fluids. US Patent 5.667.715.

Golden MA, Ulicny JC (2005) Magnetorheological fluids. US Patent 6.932.917 B2 2005.

Gonzalves FD, Koo J-H, Ahmadian M (2006) A review of the state of the art in magnetorheological fluid technologies-Part I: MR fluid and MR fluid models. Shock Vib. Dig. 38(3):203-219.

Jiles DC (1991) Introduction to magnetism and magnetic materials. Chapman \& Hall, London.

Kittipoomwong D, Klingenberg DJ, Ulicny JC (2005) Dynamic yield stress enhancement in bidisperse magnetorheological fluids. J. Rheol. 49(6):1521-1538.

Lagger HG, Breinlinger T, Korvink JG, Moseler M, Di Renzo A, Di Maio F, Bierwisch C (2015) Influence of hydrodynamic drag model on shear stress in the simulation of magnetorheological fluids. J. Non-Newtonian Fluid Mech. 218:16-26.

Lemaire E, Meunier A, Bossis G, Liu J, Felt D, Bashtovoi P, Matoussevitch N (1995) Influence of particle size on the rheology of magnetorheological fluids. J. Rheol. 39:1011-1020.

Melrose JR, Heyes DM (1993) Simulations of electrorheological and particle mixture suspensions: agglomerate and layer structures. J. Chem. Phys. 98(7): 5873-5886.

Park BJ, Fang FF, Choi HJ (2010) Magnetorheology: Materials and application. Soft Matter 6:5246-5253.

Parthasarathy M, Klingenberg DJ (1996) Electrorheology: Mechanisms and models. Mater. Sci. Eng. R. 17:57-103.

See H, Kawai A, Ikazaki F (2002) The effect of mixing particles of different size on the electrorheological response under steady shear flow. Rheol. Acta 41:55-60. 
Segovia-Gutiérrez JP, Berli CLA, de Vicente J (2012) Non-lineal viscoelasticity and two-step yielding in magnetorheology: a colloidal gel approach to understand the effect of particle concentration. J. Rheol. 56(6):1429-1448.

Segovia-Gutiérrez JP, de Vicente J, Hidalgo-Alvarez R, Puertas AM (2013) Brownian Dynamics Simulations in Magnetorheology and Comparison with Experiment. Soft Matter 9:6970-6977.

Sherman SG, Wereley NM (2013) Effect of particle size distribution on chain structures in magnetorheological fluids. IEEE Transactions on Magnetics 49(7):3430-3433.

Song KH, Park BJ, Choi HJ (2009) Effect of magnetic nanoparticle additive on characteristics of magnetorheological fluid. IEEE Trans. Mag. 45(10):4045-4048.

Tan ZJ, Zou XW, Zhang WB, Jin ZZ (1999) Influences of the size and dielectric properties of particles on electrorheological response. Phys. Rev. E 59:3177-3181.

Trendler AM, Bose H (2005) Influence of particle size on the rheological properties of magnetorheological suspensions. Int. J. Mod. Phys. B 19:1416-1422.

Wang ZW, Lin ZF, Tao RB.i (1997) Influence of the size distribution of particles on the viscous property of an electrorheological fluid. Chin. Phys. Lett. 14:151-154.

Weiss KD, Carlson JD, Nixon DA (1999) Method and magnetorheolgoical fluid formulations for increasing the output of a magnetorheological fluid device. US Patent 5.900.184.

Weiss KD, Carlson JD, Nixon DA (2000) Method and magnetorheological fluid formulations for increasing the output of a magnetorheological fluid device. US Patent 6.027.664.

Wu CW, Conrad H (1998) Influence of mixed particle size on electrorheological response. J. Appl. Phys. 83:3880. 
Table 1.- Physical characteristics of the MR fluids used in this work. $\sigma_{m}$ stands for the mean particle diameter, PDI is the polydispersity index and $v$ the standard deviation of log-normal distribution. $M_{s}$ is the saturation magnetization of the suspensions using a mixing rule.

\begin{tabular}{|c|c|c|c|c|c|c|c|}
\hline \multirow{2}{*}{ MR fluid } & \multicolumn{3}{|l|}{ Fraction in mixed suspensions (wt $)$} & \multirow{2}{*}{$\sigma_{m}(\mu \mathrm{m})$} & \multirow{2}{*}{ PDI } & \multirow{2}{*}{$\boldsymbol{N}$} & $M_{s}(\mathrm{kA} / \mathrm{m})$ \\
\cline { 2 - 5 } & HQ & HS & OM & & & & \\
\hline S & 100 & 0 & 0 & 1.26 & 1.64 & 0.42 & 1691 \\
\hline M & $\mathbf{0}$ & $\mathbf{1 0 0}$ & $\mathbf{0}$ & $\mathbf{2 . 2 0}$ & $\mathbf{1 . 6 3}$ & $\mathbf{0 . 3 8}$ & $\mathbf{1 7 0 3}$ \\
\hline L & 0 & 0 & 100 & 4.30 & 1.85 & 0.52 & 1550 \\
\hline P1 & $\mathbf{1 3}$ & $\mathbf{8 0}$ & $\mathbf{7}$ & $\mathbf{2 . 2 2}$ & $\mathbf{2 . 4 8}$ & $\mathbf{0 . 5 2}$ & $\mathbf{1 6 9 1}$ \\
\hline P2 & $\mathbf{2 0}$ & $\mathbf{7 0}$ & $\mathbf{1 0}$ & $\mathbf{2 . 2 2}$ & $\mathbf{2 . 7 1}$ & $\mathbf{0 . 5 8}$ & $\mathbf{1 6 8 5}$ \\
\hline P3 & $\mathbf{2 5}$ & $\mathbf{6 0}$ & $\mathbf{1 5}$ & $\mathbf{2 . 2 8}$ & $\mathbf{2 . 8 8}$ & $\mathbf{0 . 6 4}$ & $\mathbf{1 6 7 7}$ \\
\hline P4 & $\mathbf{3 3}$ & $\mathbf{5 0}$ & $\mathbf{1 7}$ & $\mathbf{2 . 2 5}$ & $\mathbf{3 . 0 2}$ & $\mathbf{0 . 6 8}$ & $\mathbf{1 6 7 3}$ \\
\hline P5 & $\mathbf{4 0}$ & $\mathbf{4 0}$ & $\mathbf{2 0}$ & $\mathbf{2 . 2 4}$ & $\mathbf{3 . 1 3}$ & $\mathbf{0 . 7 2}$ & $\mathbf{1 6 6 8}$ \\
\hline P6 & $\mathbf{5 0}$ & $\mathbf{3 0}$ & $\mathbf{2 0}$ & $\mathbf{2 . 1 5}$ & $\mathbf{3 . 3 1}$ & $\mathbf{0 . 7 6}$ & $\mathbf{1 6 6 6}$ \\
\hline
\end{tabular}


Table 2.- Dimensionless particle diameters, $\sigma_{s}{ }^{*}, \sigma_{a}{ }^{*}, \sigma_{b}{ }^{*}$, as a function of the polydispersity index $v$ for tri-disperse molecular dynamic simulations. Probabilities for finding particles of different sizes were fixed at $P\left(\sigma_{s}\right)=P\left(\sigma_{b}\right)=0.16$ and $P\left(\sigma_{a}\right)=0.68$

\begin{tabular}{|c|c|c|c|}
\hline$v$ & $\sigma_{\mathrm{s}}{ }^{*}$ & $\sigma_{\mathrm{a}}{ }^{*}$ & $\sigma_{\mathrm{b}}{ }^{*}$ \\
\hline 0.38 & 0.567 & 1.021 & 1.713 \\
\hline 0.52 & 0.464 & 1.040 & 2.277 \\
\hline 0.58 & 0.425 & 1.050 & 2.515 \\
\hline 0.64 & 0.391 & 1.061 & 2.780 \\
\hline 0.72 & 0.350 & 1.077 & 3.183 \\
\hline 0.76 & 0.330 & 1.086 & 3.409 \\
\hline
\end{tabular}


Figure 1. Particle size distributions for the polydisperse MR fluids investigated in experiments.

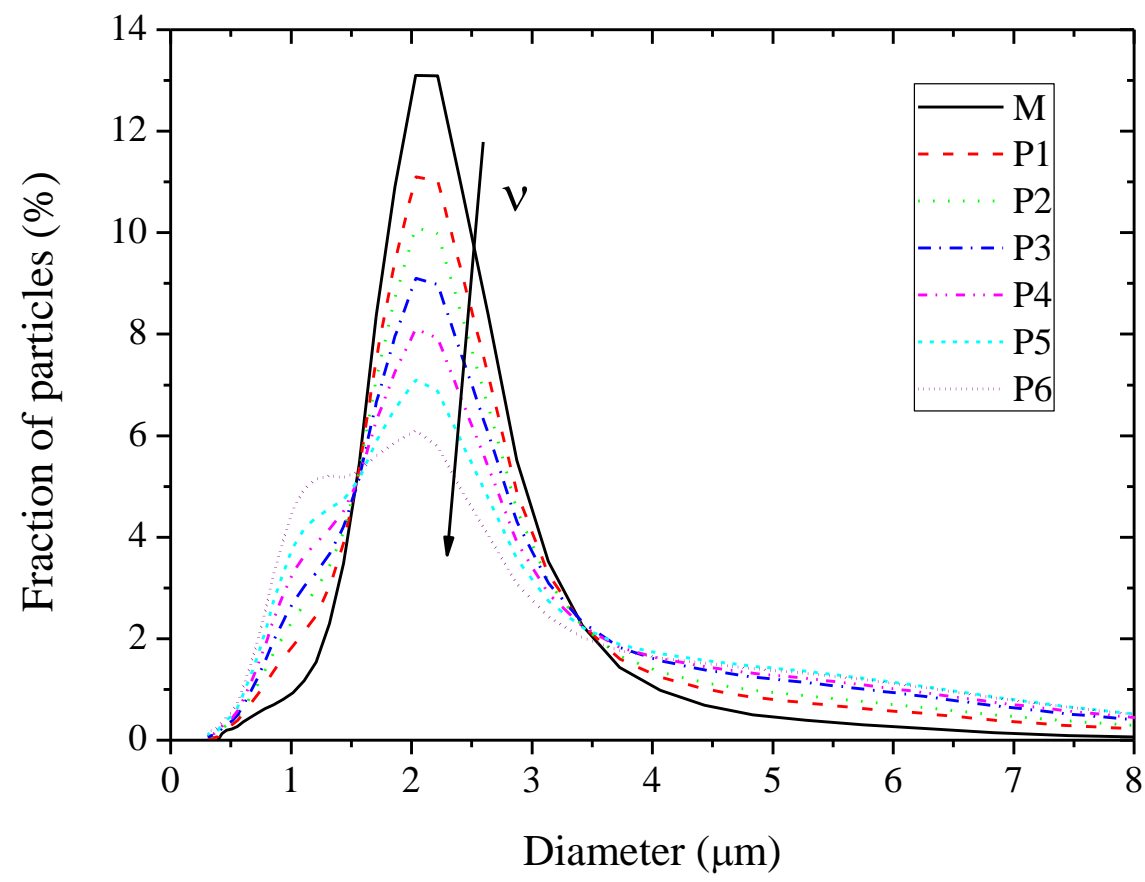


Figure 2. Experimental rheograms (shear stress versus shear rate) for different polydispersity levels in $\phi=0.10 \mathrm{MR}$ fluids. The shear stress window corresponds to the range $4-12 \mathrm{kPa}$.

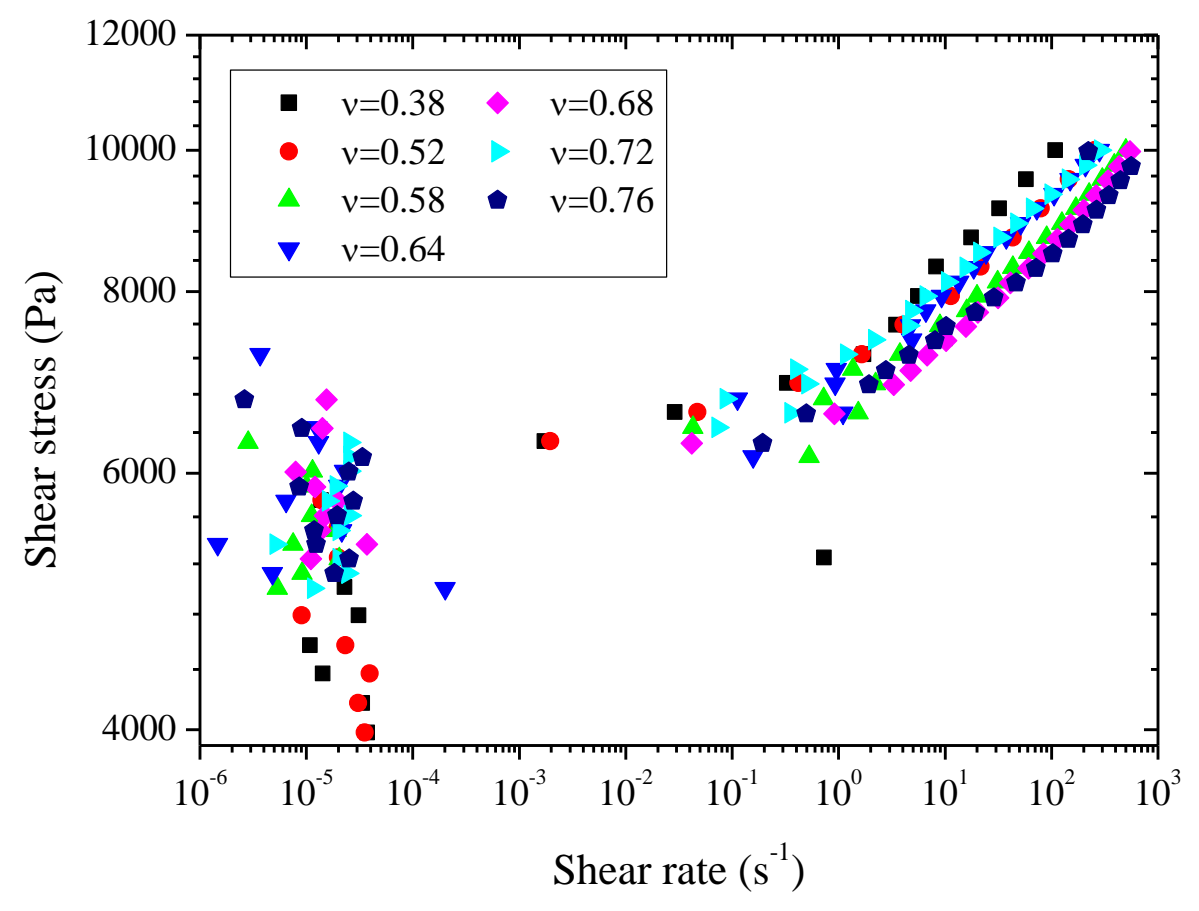


Figure 3. Experimental static and dynamic apparent yield stresses as a function of polydispersity index $v$ for $\phi=0.10$ MR fluids.

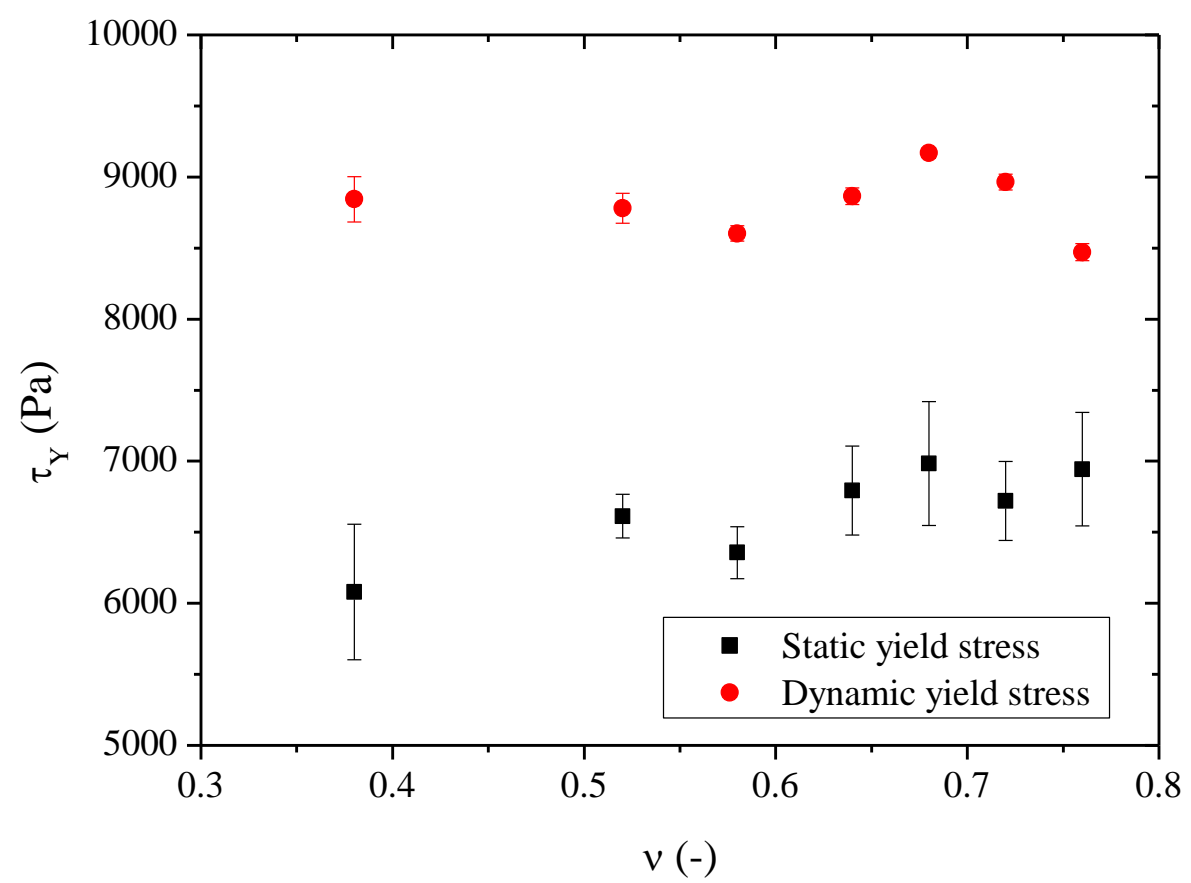


Figure 4. Typical example of the simulated shear stress-strain curves for $\phi=0.10$ and $v=0.64$. Every curve corresponds to an independent simulation run. a) Tests up to strain $\gamma=1$. b) Tests up to $\gamma=3$. Dimensionless shear rate is $\dot{\gamma}^{*}=10^{-3}$.
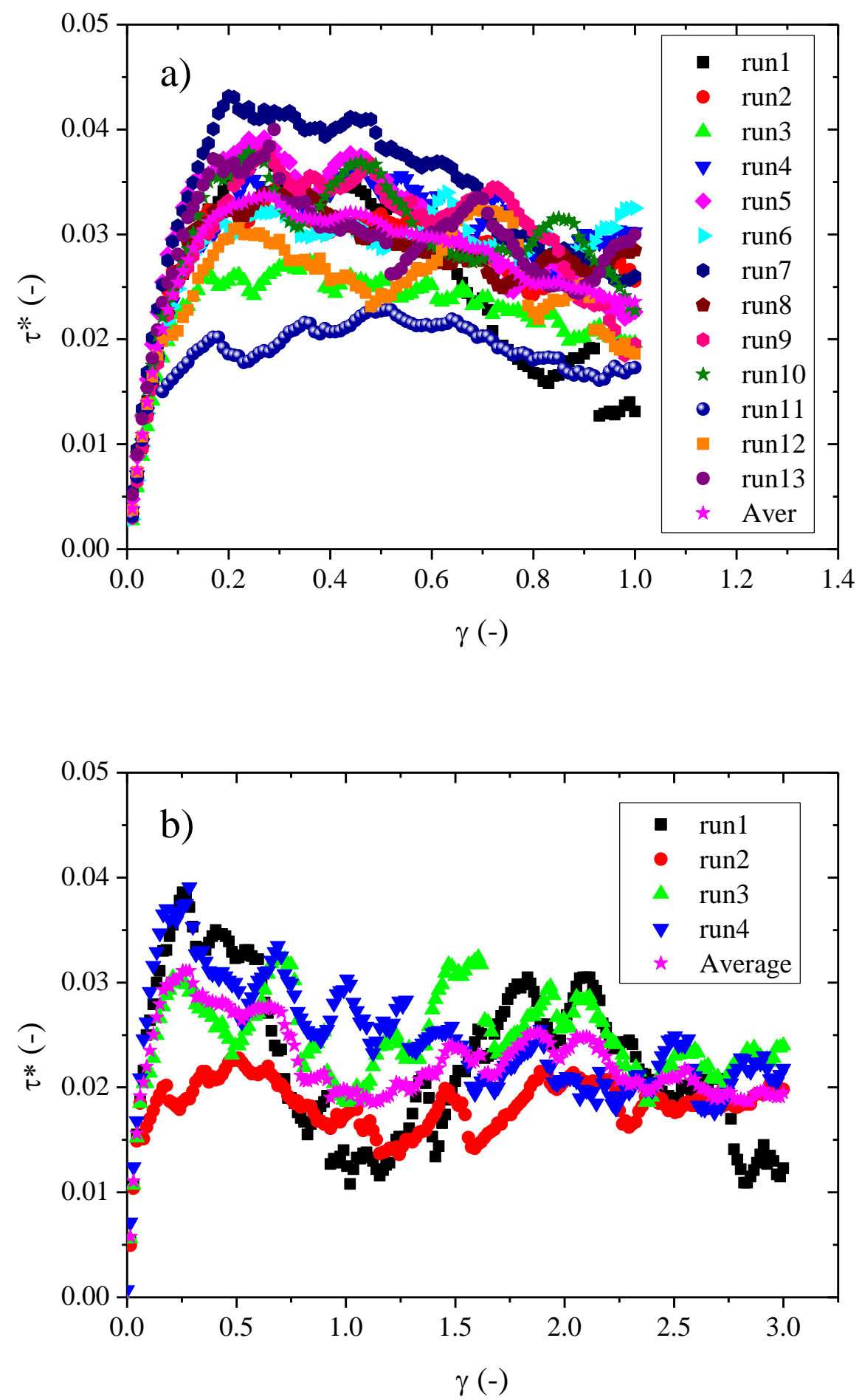
Figure 5. Experimental and simulation static and dynamic yield stresses as obtained from the maximum and long-time plateau in stress-strain curves reported in Figure 4 for different polydispersity indexes in the $\phi=0.10$ MR fluids. Squares correspond to continuous particle size distributions. Circles correspond to tri-disperse distributions. Triangles correspond to experimental data.

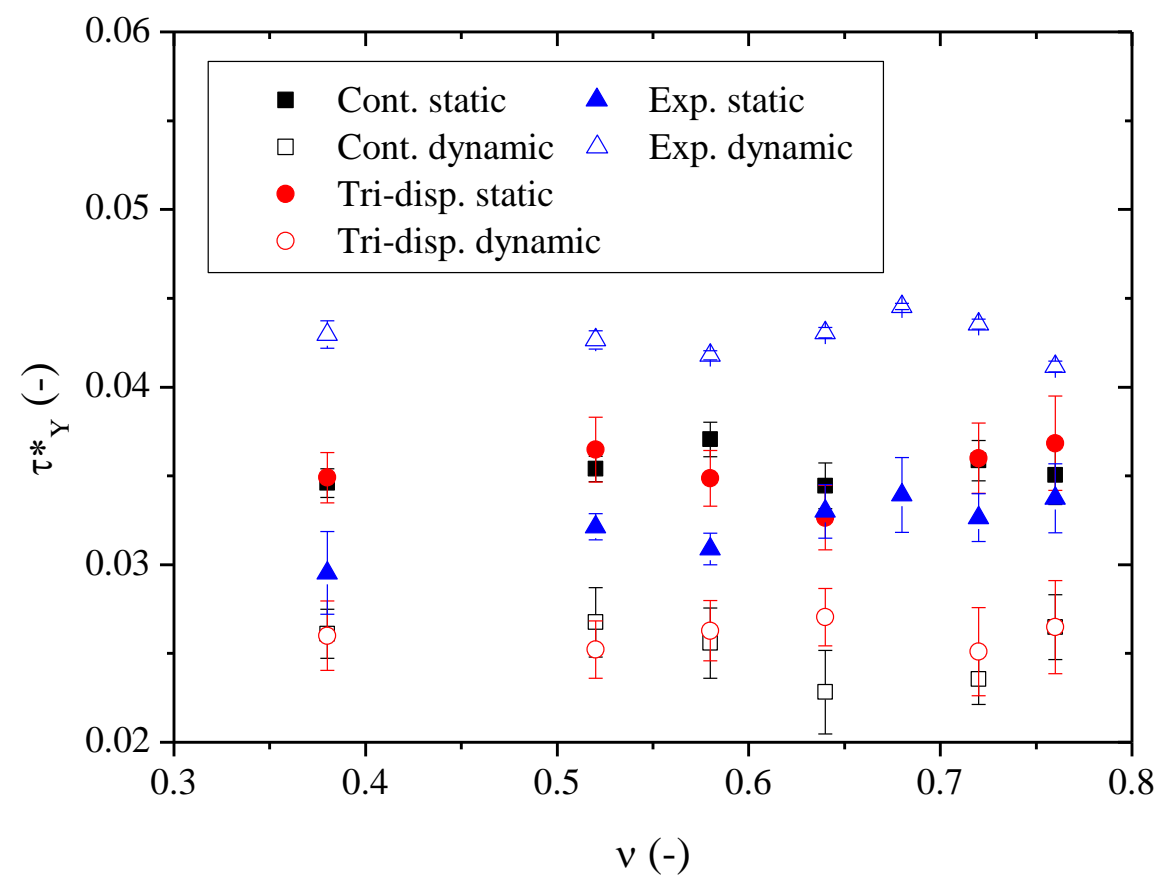


Figure 6. Simulation results on the average number of particles per cluster, $N_{p c}$, for MR fluids with continuous particle size distributions at fixed particle concentration $\phi=0.10$. a) Prior to shear; b) Under shearing.
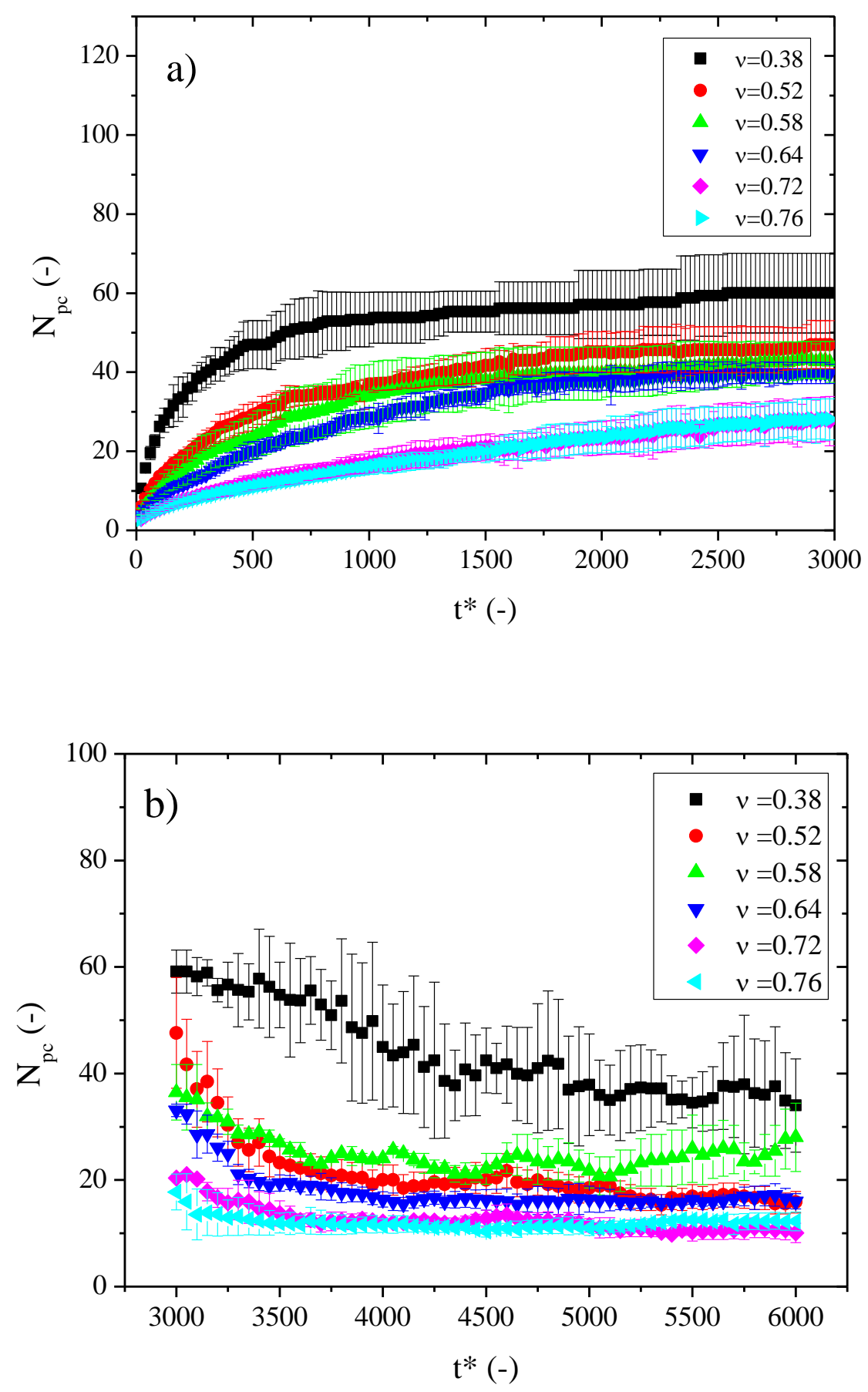
Figure 7.- Particle radial distribution function in MR fluids with continuous particle size distributions and $\phi=0.10$ at three different stages of the deformation: a) elastic region (at $\gamma=0.1$ ), b) stress peak, c) steady region. The results in the steady region were calculated by averaging over 4000 configurations saved from $\gamma=2$ to $\gamma=3$.
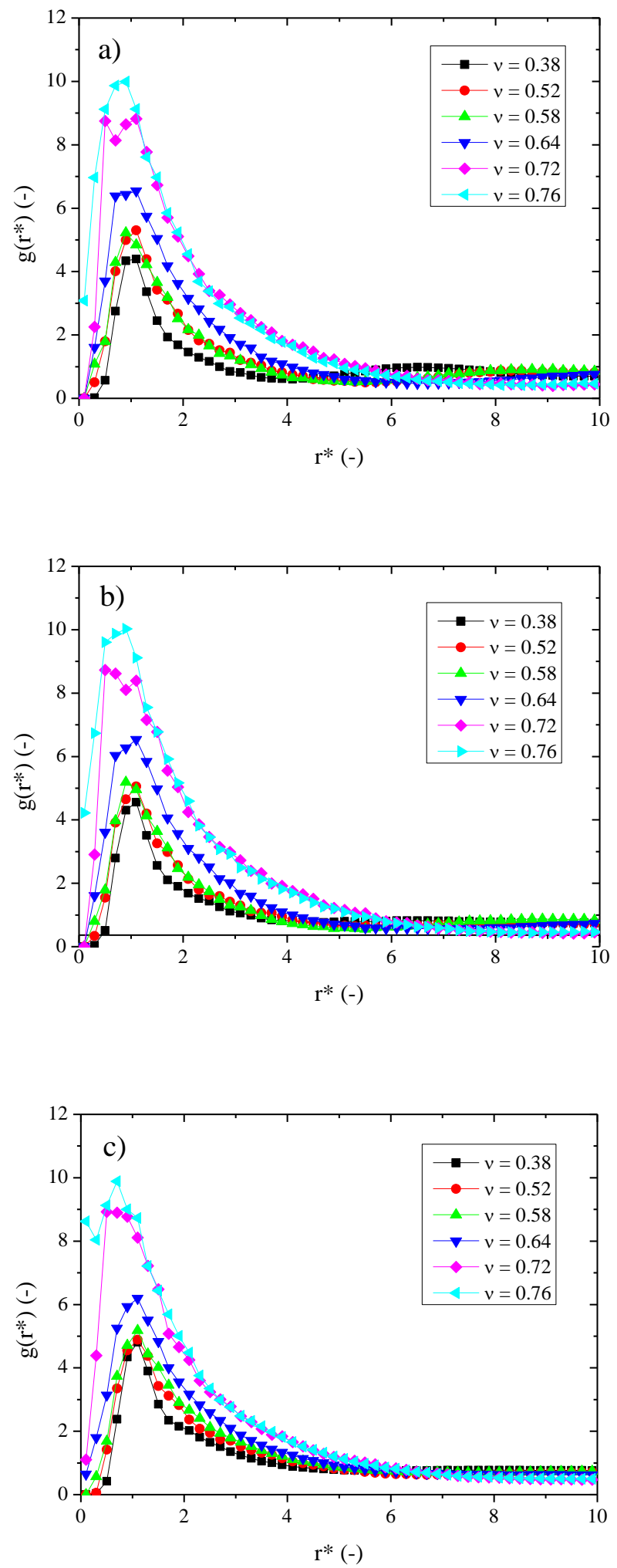
Figure 8.- Polydispersity dependence of the maximum value of the pair distribution function, $g(r, \theta)$, averaged within different angle limits for the $\phi=0.10$ MR fluids.

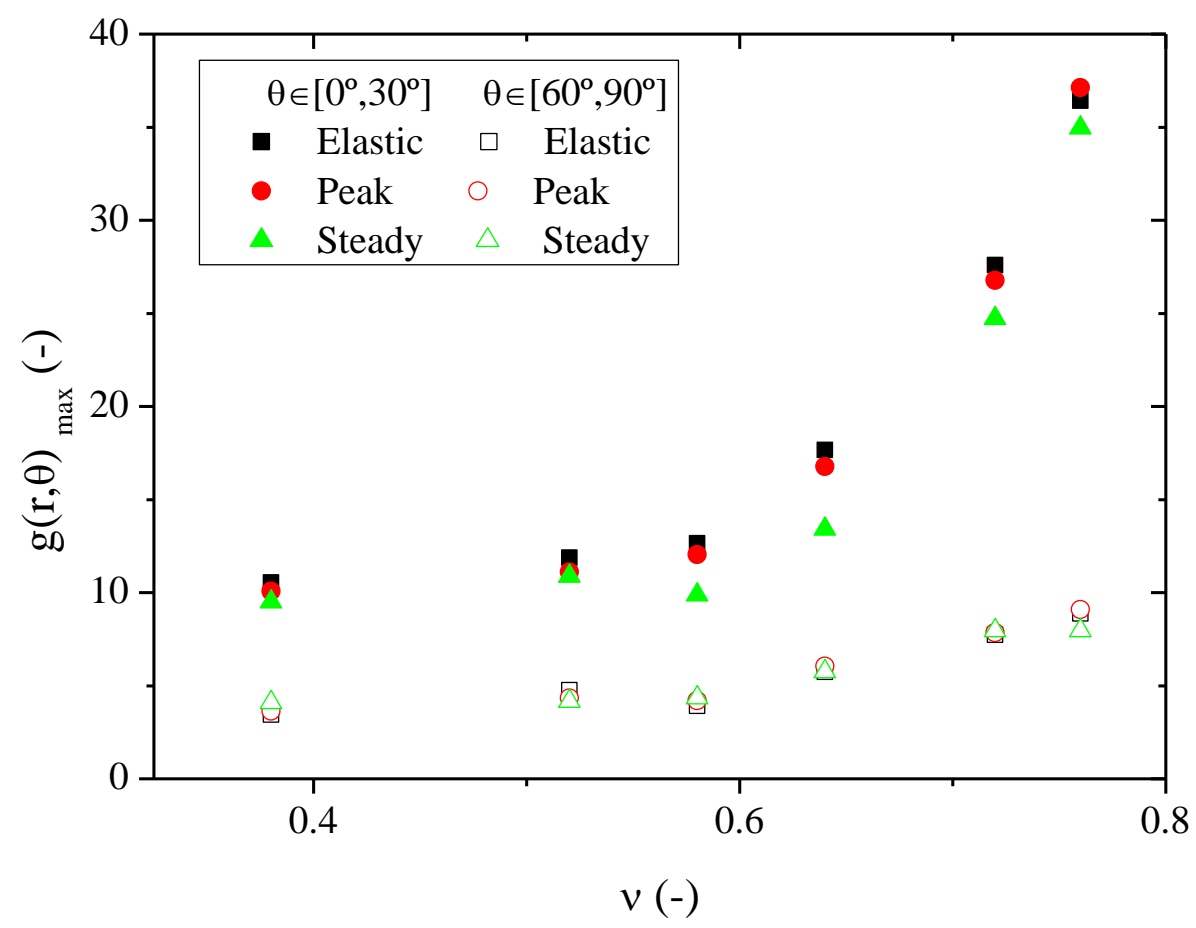


Figure 9.- Radial distribution function for tri-disperse particle size distributions. $\phi=0.10$. a) elastic region, b) stress peak, c) steady region. The steady region was calculated by averaging over 4000 configurations from $\gamma=2$ to $\gamma=3$.
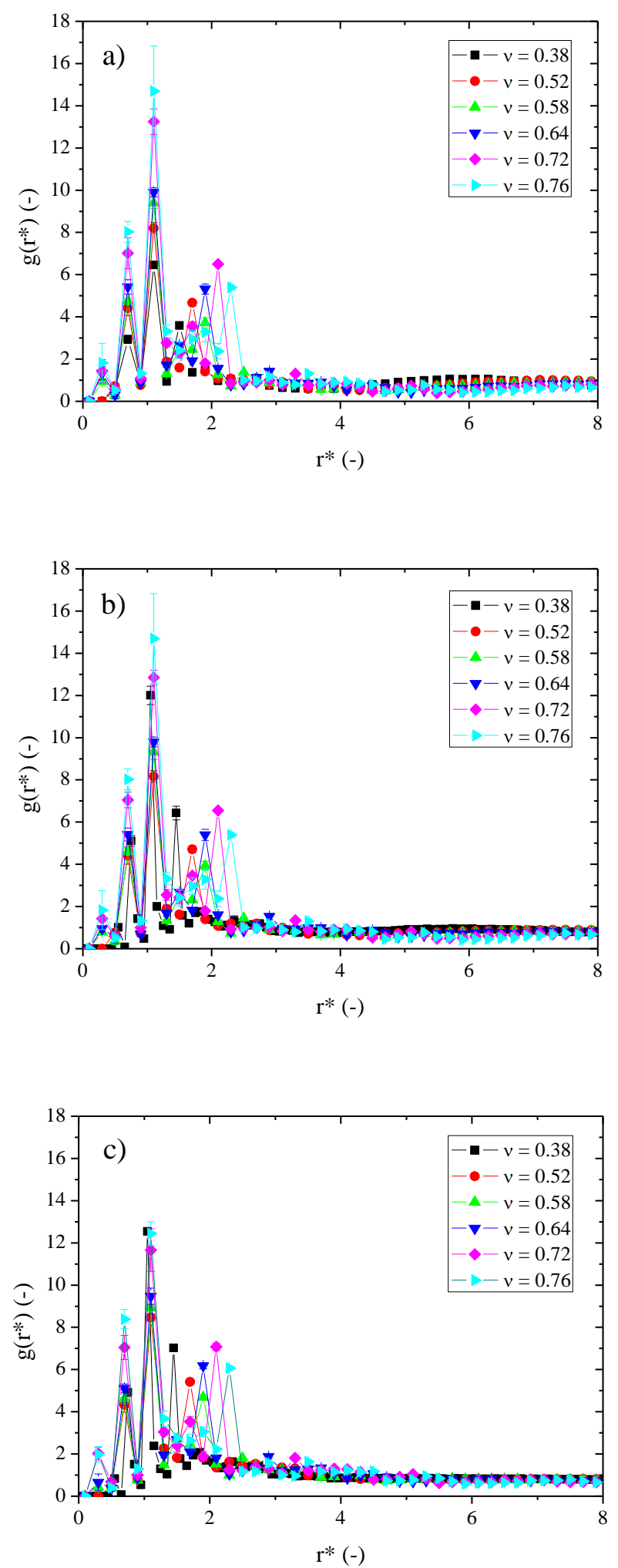
Figure 10.- Snapshots of the microstructure prior to shear for $\phi=0.10$ : a) $v=0.52$ and b) $v=0.72$.

a)

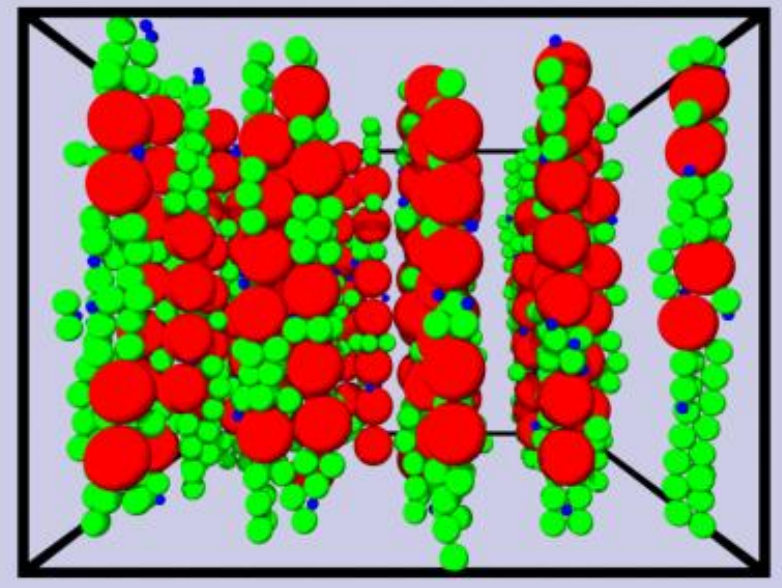

b)

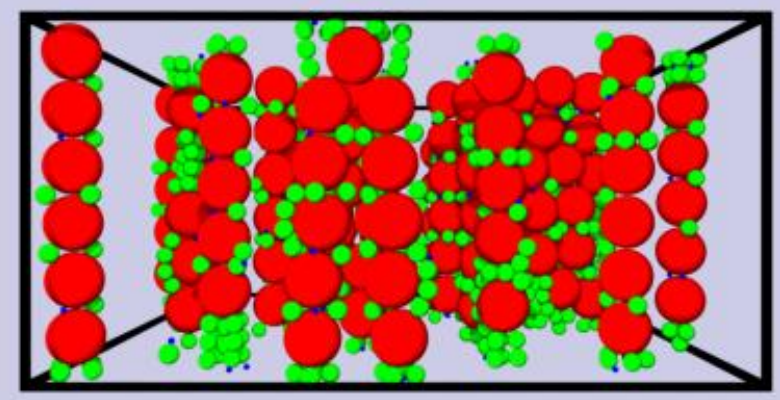


Figure 11.- Peak heights of the radial distribution functions in tri-disperse simulations as a function of the polydispersity index. The particle concentration is $\phi=0.10$. a) elastic region, b) peak region and c) steady region. Normalization of the peak heights by the probabilities: d) elastic region, e) peak region and f) steady region.
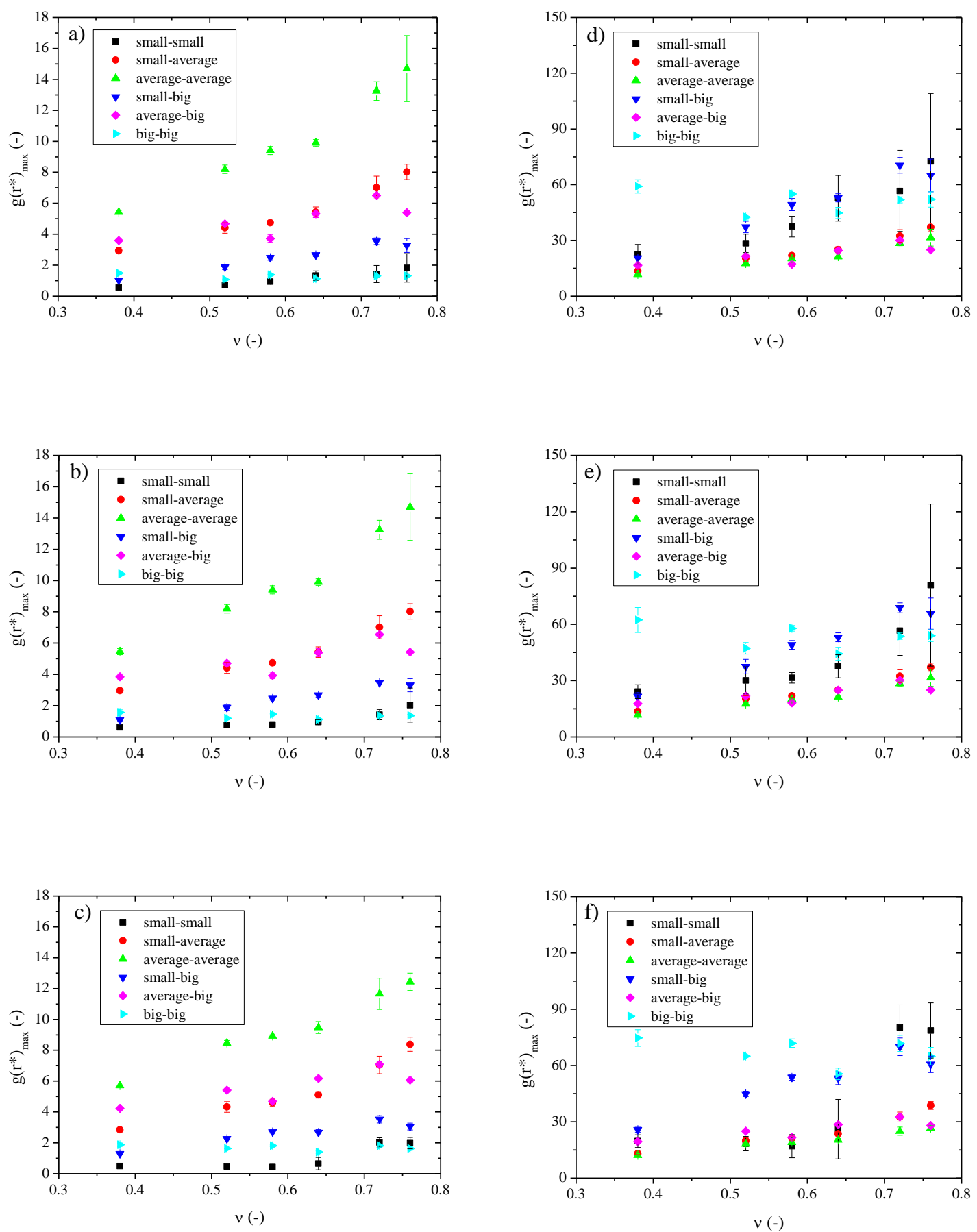


\section{Supporting information}

Figure S1.- Experimental static yield stress and the dynamic yield stress for an MR fluid with $v=0.76$ and $\phi=0.10$.

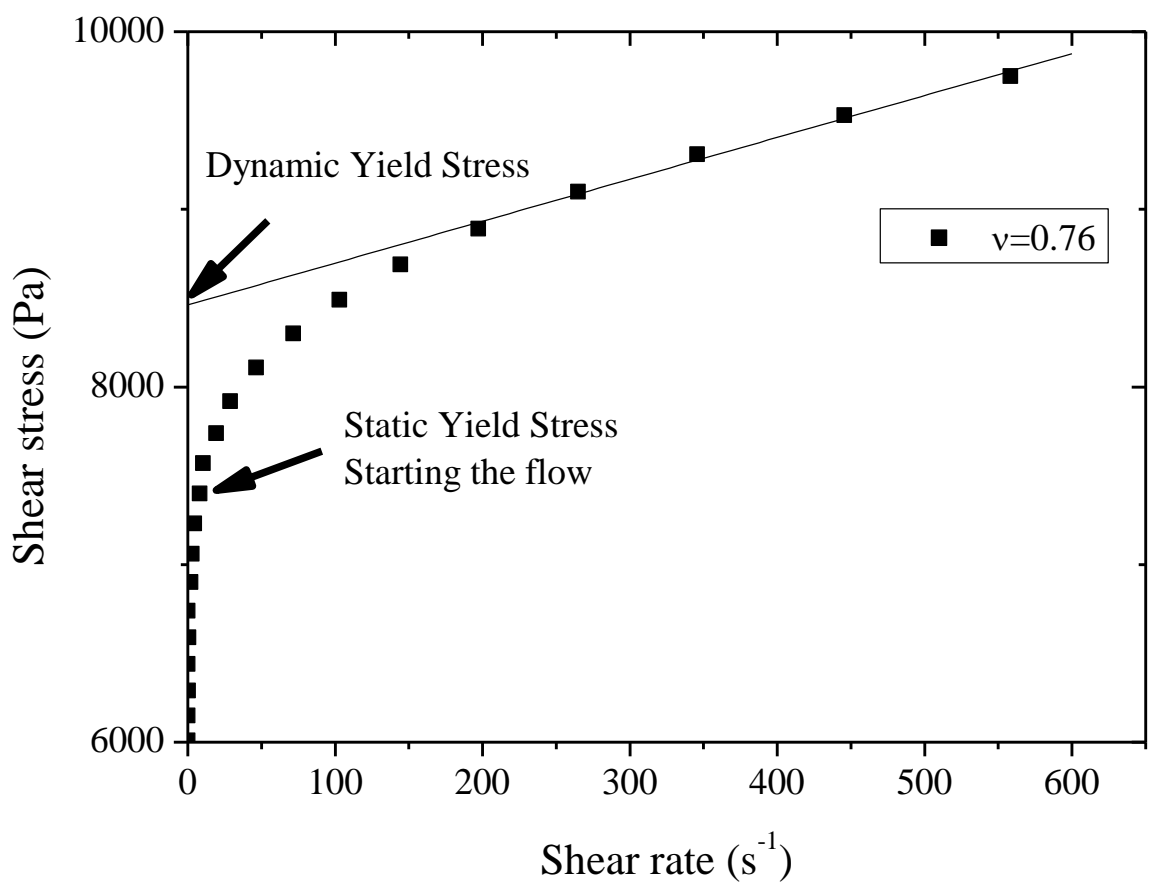


Figure S2.- Simulation results on the average number of cluster, $N_{c}$ for MR fluids with continuous particle size distributions at particle concentration $\phi=0.10$. a) prior to shear, b) under shearing. The total number of particles in the simulation box is $N=1000$.
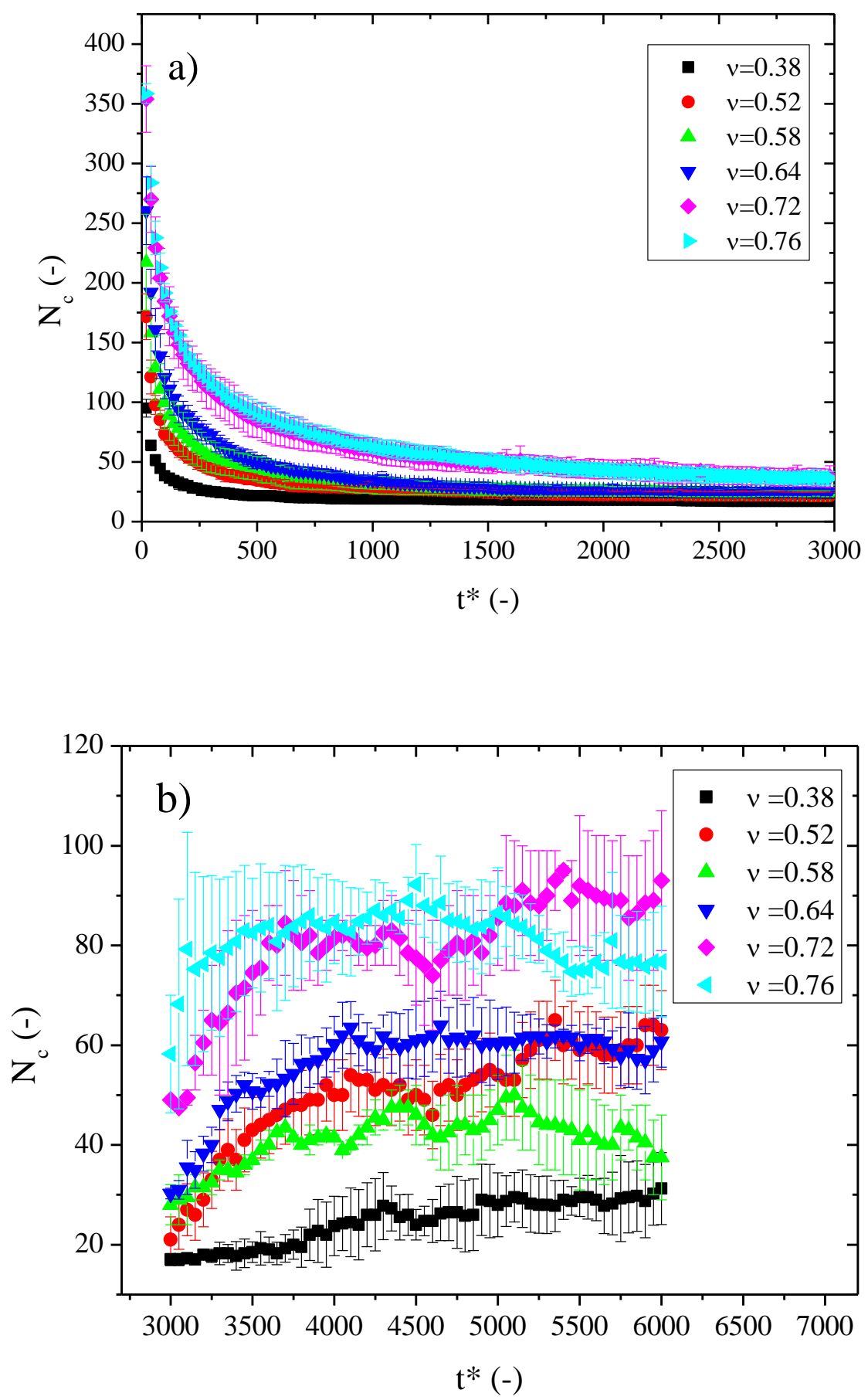
Figure S3.- Simulation results on the weight-averaged number of particles in each cluster $S_{2}$ for MR fluids with continuous particle size distributions at fixed particle concentration $\phi=0.10$. a) prior to shear; b) under shearing.
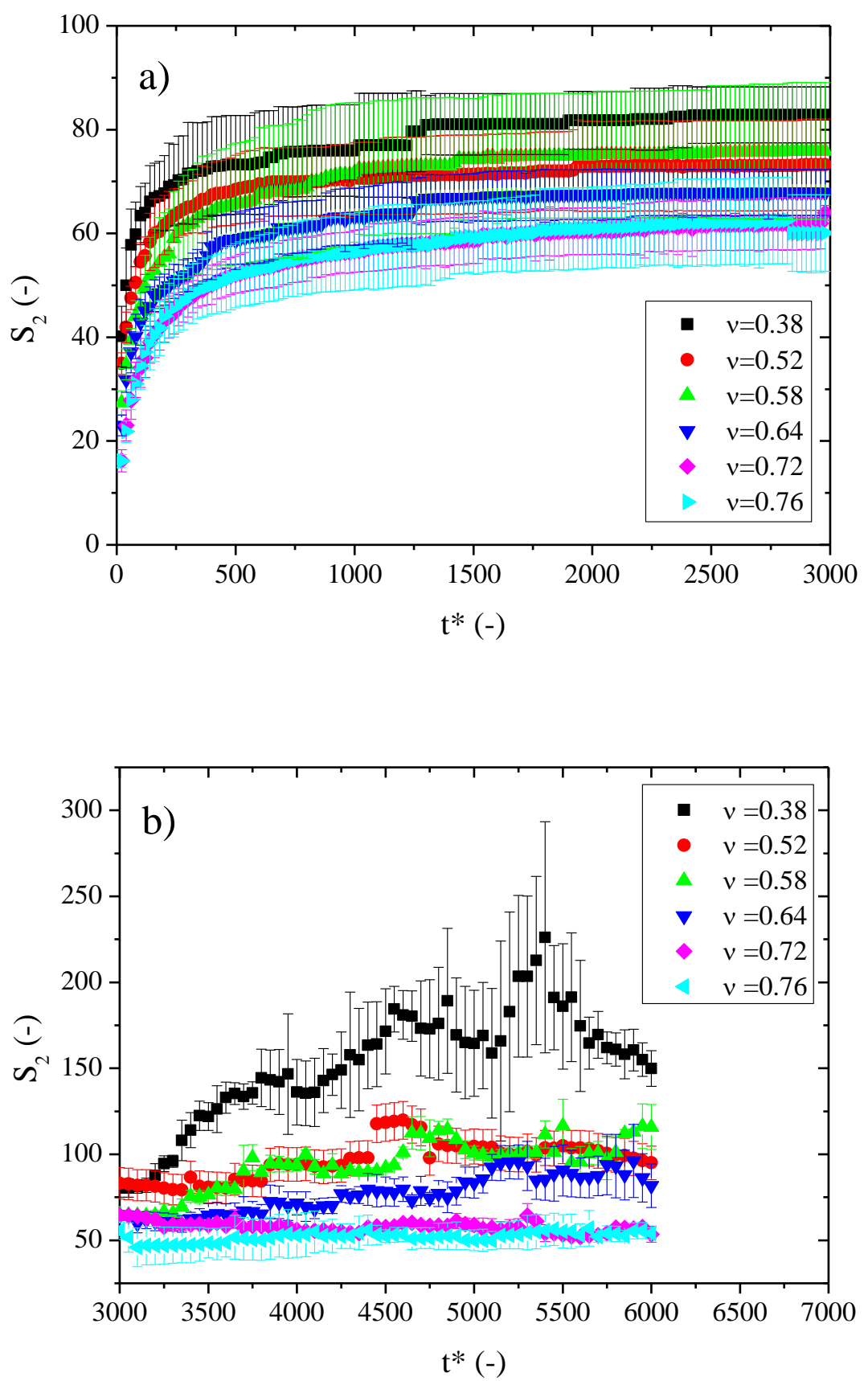